\title{
ABOUT SUBSTITUTION TILINGS WITH STATISTICAL CIRCULAR SYMMETRY
}

\author{
D. FRETTLÖH
}

\begin{abstract}
Two results about equidistribution of tile orientations in primitive substitution tilings are stated, one for finitely many, one for infinitely many orientations. Furthermore, consequences for the associated diffraction spectra and the dynamical systems are discussed.
\end{abstract}

\section{Substitution tilings}

Several important models of quasicrystals are provided by (decorations of) nonperiodic substitution tilings. In this paper, only tilings of the plane are considered. In larger dimensions some aspects become rather complicated, in particular, generalisations of Theorem 2.2. A substitution (or tile-substitution, but we stick to the shorter term here) is just a rule how to enlarge and dissect a collection of tiles $P_{1}, \ldots, P_{m}$ into copies of $P_{1}, \ldots, P_{m}$, as indicated in Figure 1 (left) or Figure 2, Iterating this rule yields tilings of larger and larger portions of the plane. However, the precise description of a substitution tiling in $\mathbb{R}^{2}$ is usually given as follows.

Let $P_{1}, \ldots, P_{m}$ be compact subsets of $\mathbb{R}^{2}$, such that each $P_{i}$ equals the closure of its interior. These are the prototiles, the pieces our tiling is built of. We refer to sets which are congruent to some prototile simply as tiles. (Sometimes two prototiles are allowed to be congruent, compare Figure 1. Then we equip each one of them with an additional attribute (colour, decoration) to distinct them. Whenever we speak of 'congruent' tiles in the sequel, this means 'congruent with respect to possible decorations'.) A tiling (with prototiles $P_{1}, \ldots, P_{m}$ ) is a collection of tiles (each one congruent to some $P_{i}$ ) which covers $\mathbb{R}^{2}$ such that every $x \in \mathbb{R}^{2}$ is contained in the interior of at most one tile. Note, that each such tiling $\mathcal{T}$ can be described by placements of the prototiles:

$$
\mathcal{T}=\left\{R_{\alpha_{1}} P_{i_{1}}+t_{1}, R_{\alpha_{2}} P_{i_{2}}+t_{2}, \ldots\right\},
$$

where $R_{\alpha_{i}}$ denotes a rotation through $\alpha_{i}$, and $t_{i} \in \mathbb{R}^{2}$ are translation vectors. For later purposes, we define the orientation $\phi(T)$ of a tile: If $T=R_{\alpha} P_{i}+t, \alpha \in[0,2 \pi[$, then $\phi(T)=\alpha$.

Now, let $\lambda>1$ and $\sigma$ be a map which maps each prototile $P_{i}$ to a non-overlapping collection of tiles, each one congruent to some prototile $P_{i}$. Furthermore, we require the substitution $\sigma$ to be selfsimilar in the sequel. That is, $\lambda P_{i}=\bigcup_{T \in \sigma\left(P_{i}\right)} T$ for $1 \leq i \leq m$. By setting $\sigma\left(R P_{i}+t\right)=R \sigma\left(P_{i}\right)+\lambda t$, the substitution $\sigma$ extends naturally to any tile congruent to some prototile. In a similar way, by $\sigma\left(\left\{T_{1}, T_{2}, \ldots\right\}\right)=\sigma\left(T_{1}\right) \cup \sigma\left(T_{2}\right) \cup \ldots$, the substitution $\sigma$ extends to all (finite or infinite) collections of tiles. In particular, one can iterate $\sigma$ on the prototiles to obtain the $k$-th order supertiles $\sigma^{k}\left(P_{i}\right)$.

Definition 1.1. Let $\sigma$ be a tile-substitution with prototiles $P_{1}, \ldots, P_{m}$. A tiling $\mathcal{T}$ is called substitution tiling (with substitution $\sigma$ ) if for each finite subset $\mathcal{F} \subset \mathcal{T}$ there are $i, k$ such that a copy of $\mathcal{F}$ is contained in some supertile $\sigma^{k}\left(T_{i}\right)$. The family of all substitution tilings with 
substitution $\sigma$ is denoted by $\mathbb{X}_{\sigma}$. The matrix $M_{\sigma}$ with $\left(M_{\sigma}\right)_{i j}=\#\left\{T \in \sigma\left(P_{j}\right): T=R P_{i}+t\right\}$ is called substitution matrix. A substitution is primitive, if there is $k \geq 1$ such that $M_{\sigma}^{k}$ is strictly positive.

Here, $\# A$ denotes the cardinality of the set $A$. This definition of a substitution tiling fits well into several contexts, for instance, to the hull of a tiling, see Section 4 .

\section{Statistical CIRCUlar Symmetry}

Most of the well-known substitution tilings have the property that all prototiles occur in only finitely many orientations. For instance, the Penrose dart and kite tiling has just two prototiles, a convex and a non-convex quadrangle. Each one occurs in one of 10 different orientations throughout the tiling. There are also substitution tilings where the tiles occur in infinitely many orientations. The most prominent examples are certainly the pinwheel tilings [14. But there are many other examples, compare [6], [7]. Two examples are shown in Figure 2 .

It was known for the pinwheel tiling that there are not only infinitely many orientations of the tiles, but that they are furthermore equidistributed [14, [12. Intuitively, this means that each orientation occurs with the same frequency throughout the tiling. In order to make this precise, we need the following definition. Recall that a sequence $\left(a_{j}\right)_{j \geq 0}$ is equidistributed in $[0,2 \pi[$, if for all $0 \leq x<y<2 \pi$ holds:

$$
\lim _{n \rightarrow \infty} \frac{1}{n} \sum_{j=1}^{n} 1_{[x, y]}\left(a_{j}\right)=\frac{x-y}{2 \pi} .
$$

Since the above sum is not absolutely convergent, it depends strongly on the ordering of the sequence. Thus we need to order the tiles in the tiling in some not-too-weird way. This is done in the following definition.

Definition 2.1. Let $\mathcal{T}=\left\{T_{1}, T_{2}, \ldots\right\}$ be a primitive substitution tiling. Let the numbering be such that the sequence $\left(T_{j}\right)_{j \geq 1}$ satisfies the following property: for all $n \geq 1$, there is some $\ell \geq n$ such that the patch $\left\{T_{1} \ldots, T_{\ell}\right\}$ is congruent to some supertile $\sigma^{k}\left(P_{i}\right)$ for some prototile $P_{i}, k \geq 1$. The tiling $\mathcal{T}_{\sigma}$ has statistical circular symmetry, if, for all $0 \leq x<y<2 \pi$, one has:

$$
\lim _{r \rightarrow \infty} \frac{1}{n} \sum_{j=1}^{n} 1_{[x, y]}\left(\phi\left(T_{j}\right)\right)=\frac{x-y}{2 \pi} .
$$

The ordering in the definition is compatible with other natural notions, for instance, ordering the tiles with respect to their distance to the origin. The following result was obtained in 6]. Only while preparing the present text, the author realized that a similar result already appeared in [14]; see also the comments in Section 4.

Theorem 2.2. Let $\mathcal{T}$ be a primitive substitution tiling in $\mathbb{R}^{2}$, where copies of some prototile occur in infinitely many orientations. Then $\mathcal{T}$ is of statistical circular symmetry.

In the next section this theorem is used to show that any such tiling has circular diffraction spectrum. Now we show an analogue of the theorem above for tilings with tiles in finitely many orientations. One can ask whether there are substitution tilings where tiles occur more frequent in one direction than the other. This is possible, see Figure 1 for an example. But this is achieved by substituting the horizontal rectangle differently from the vertical rectangle. 

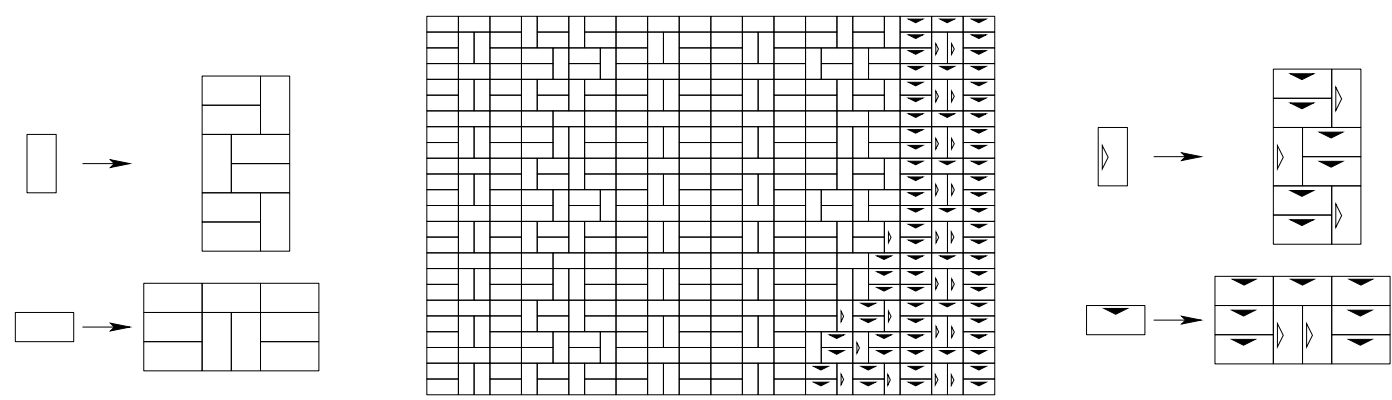

FiguRE 1. A substitution tiling where horizontal rectangles are more frequent in the tiling than vertical ones. By Theorem 2.3 , this can be achieved only when the two types of rectangles are substituted differently from each other.

In fact we have two prototiles in this example, each one occurring in one direction only. This is illustrated in the right part of Figure 1 by assigning different decorations to the tiles. The following theorem states that each prototile $P_{i}$ occurs in each of its orientations with the same frequency in a primitive substitution tiling. In order to make this precise, let freq $\left(P_{i}, \alpha\right)$ denote the frequency of tiles $R_{\alpha} P_{i}+t$ in a tiling $\mathcal{T}$. That is,

$$
\operatorname{freq}\left(P_{i}, \alpha, \mathcal{T}\right)=\lim _{r \rightarrow \infty} \frac{\#\left\{T \in \mathcal{T} \cap B_{r}: \exists t T=R_{\alpha} P_{i}+t\right\}}{\#\left\{T \in \mathcal{T} \cap B_{r}\right\}}
$$

where $B_{r}$ denotes the closed ball of radius $r$ around the origin. For primitive substitution tilings, this frequency exists and is independent of the choice of the origin. This can be seen as follows: Up to now, we identified types of tiles with respect to congruence congruence classes. In a tiling with finitely many orientations, one can as well identify types of tiles with respect to translation classes. Usually, this results in a larger number of prototiles (e.g., the Penrose dart and kite tilings have two prototiles up to congruence, but 20 prototiles up to translation). The frequency of each prototile in a primitive substitution tiling can be obtained from the substitution matrix $M_{\sigma}$. This result is folklore, see for instance [13, [5]: The normed Perron-Frobenius eigenvector of $M_{\sigma}$ contains the relative frequencies of the prototiles. In particular, in all tilings in $\mathbb{X}_{\sigma}$, these frequencies are the same. Therefore, for any given primitive substitution, we can drop the $\mathcal{T}$ in the definition of the frequency in this case and write just freq $\left(P_{i}, \alpha\right)$.

Theorem 2.3. Let $\sigma$ be a primitive substitution with prototiles $\left\{P_{1}, \ldots, P_{m}\right\}$. If freq $\left(P_{i}, \alpha\right) \neq$ 0 , freq $\left(P_{i}, \beta\right) \neq 0$ then $\operatorname{freq}\left(P_{i}, \alpha\right)=\operatorname{freq}\left(P_{i}, \beta\right)$.

Proof. If freq $\left(P_{i}, \alpha\right) \neq 0$, then the prototiles occur in finitely many orientations only, by primitivity of the substitution and Theorem 2.2. Therefore we obtain finitely many prototiles with respect to translations: $\left(P_{1}, 0\right),\left(P_{1}, \alpha_{1,1}\right),\left(P_{1}, \alpha_{1,2}\right), \ldots,\left(P_{1}, \alpha_{1, k}\right) ;\left(P_{2}, 0\right),\left(P_{2}, \alpha_{2,1}\right), \ldots \ldots\left(P_{m}, \alpha_{m, \ell}\right)$.

Consider a tiling $\mathcal{T} \in \mathbb{X}_{\sigma}$. By the remarks preceding the theorem, freq $\left(P_{i}, 0\right)$ is well defined, and its value is the same in the tiling $\mathcal{T}$ and in the tiling $R_{\alpha_{i, j}} \mathcal{T} \in \mathbb{X}_{\sigma}$. And trivially, freq $\left(P_{i}, 0\right)$ in $\mathcal{T}$ equals freq $\left(P_{i}, \alpha_{i, j}\right)$ in $R_{\alpha_{i, j}} \mathcal{T}$. Therefore, freq $\left(P_{i}, 0\right)=\operatorname{freq}\left(P_{i}, \alpha_{i, j}\right)$, and the claim follows. 



Figure 2. Two substitutions yielding tiling with statistical circular symmetry. The left one is due to Danzer and Goodman-Strauss [8], the right one is from [6].

\section{Diffraction}

There is a wealth of literature devoted to the mathematical description of the diffraction spectrum of nonperiodic structures, see for instance [4], 9], [10, [18], or the introductory text [1] and references therein. Usually one starts with a discrete point set $\Lambda \subset \mathbb{R}^{2}$ rather than a tiling. In the present context of tilings, this can be achieved by decorating each prototile with one or more points ('atomic decorations').

Let $\Lambda$ be such a point set, obtained from a tiling. The diffraction spectrum of $\Lambda$ is the Fourier transform of the autocorrelation of $\Lambda$. The autocorrelation (also called Patterson function) of $\Lambda$ is

$$
\gamma=\gamma_{\Lambda}=\lim _{r \rightarrow \infty} \frac{1}{\operatorname{vol}\left(B_{r}\right)} \sum_{x, y \in \Lambda \cap B_{r}} \delta_{x-y},
$$

if this limit exists. Here and in the following we are working in the framework of tempered distributions [17, [19]. Thus the limit above is to be understood as a vague limit. If the limit does not exist, there is a subsequence converging to an autocorrelation which is known to be translation bounded (since we started with a translation bounded measure $\delta_{\Lambda}$ ) and positive definite. Thus $\gamma$ can be regarded as a translation bounded positive definite measure. Now, the Fourier transform $\widehat{\gamma}$ of $\gamma$ is the diffraction measure, and its support is the diffraction spectrum of $\Lambda$. We are interested in the nature of $\widehat{\gamma}$. By Lebesgue's decomposition theorem, $\widehat{\gamma}$ has a unique decomposition

$$
\widehat{\gamma}=\widehat{\gamma}_{p p}+\widehat{\gamma}_{s c}+\widehat{\gamma}_{a c}
$$

with respect to the Lebesgue measure in $\mathbb{R}^{2}$. The pure point part $\widehat{\gamma}_{p p}$ is a countable sum of Dirac measures. The absolutely continuous part $\widehat{\gamma}_{a c}$ is a measure with locally integrable density function supported on a set of positive Lebesgue measure. The singular continuous $\widehat{\gamma}_{s c}$ part assigns zero to all finite sets, but is supported on a set with Lebesgue measure zero. The (idealized) diffraction spectrum of a quasicrystal is pure point, the singular parts vanish completely. 
The following results show that substitution tilings with statistical circular symmetry have a diffraction measure which is of (perfect) circular symmetry. Consequently, the pure point part of the diffraction is trivial.

Theorem 3.1. Let $\mathcal{T}=\left\{R_{\alpha_{1}} P_{i_{1}}+t_{1}, R_{\alpha_{2}} P_{i_{2}}+t_{2}, \ldots\right\}$ be a primitive substitution tiling of statistical circular symmetry, with prototiles $P_{1}, \ldots, P_{m}$. Choose 'control points' $x_{i} \in P_{i}$, and define $\Lambda=\left\{R_{\alpha_{1}} x_{i_{1}}+t_{1}, R_{\alpha_{2}} x_{i_{2}}+t_{2}, \ldots\right\}$. Then the autocorrelation $\gamma_{\Lambda}$ of $\Lambda$ is circular symmetric.

The subtlety of this theorem is that the autocorrelation is not of statistical circular symmetry only (this is easy to see) but of perfect circular symmetry. The rotated measure $R . \mu$ is defined by $R . \mu(A)=\mu\left(R^{-1} A\right)$ for all measurable sets $A$. Then $\mu$ is of circular symmetry if $R . \mu=\mu$ for all $R$.

Proof. Since $\mathcal{T}$ is of statistical circular symmetry, $\mathcal{T}$ contains tiles $T_{j}$ congruent to some prototile $P_{i}$ for (countably) infinitely many distinct angles $\alpha_{j}=\phi\left(T_{j}\right)$. By equidistribution, the frequency of tiles in one certain orientation is 0 . But, again by the equidistribution of $\left(\alpha_{j}\right)_{j \geq 0}$, the frequency of tiles in a certain range of orientations - say, $[\beta, \gamma[\subseteq[0,2 \pi[-$ equals the frequency of tiles in orientations $[\beta+c, \gamma+c[\bmod 2 \pi$ for all $c$. The same is obviously true for supertiles in $\mathcal{T}$.

The autocorrelation is defined via difference vectors $x-y$. We deduce the circular symmetry of the autocorrelation by assigning these vectors $x-y$ to supertiles in $\mathcal{T}$. Each such vector arises from a constellation of two tiles (usually not adjacent). Consider only vectors $x-y$ of fixed a length $r>0$. Let $k \geq 1$. Then either $x$ and $y$ are contained in the same $k$-th order supertile, or in two different $k$-th order supertiles. With growing $k$, the ratio of the latter kind to the former kind tends to zero. Thus it suffices to regard the former kind only. Each one arises from a $k$-th order supertile, these are equidistributed, hence equidistribution of orientations of difference vectors $x-y$ of radius $r$ follows. In other words, the frequency of difference vectors $x-y$ of length $r$ with orientation $\varphi \in[\beta, \gamma[\subseteq[0,2 \pi$ [ equals the frequency of difference vectors of length $r$ in orientations $[\beta+c, \gamma+c[\bmod 2 \pi$ for all $c$. It follows that $\gamma_{\Lambda}=R \cdot \gamma_{\Lambda}$ for all rotations $R$.

Now we can apply the following standard result, which can be found for instance in [2].

Lemma 3.2. Let $R$ be an orthogonal map, $\mu$ a measure, and let the measure $R$. $\mu$ be given by $R . \mu(A)=\mu\left(R^{-1} A\right)$. Then $R . \widehat{\mu}=\widehat{R . \mu}$.

Since $R \cdot \gamma_{\Lambda}=\gamma_{\Lambda}$, with $\Lambda$ as in 3.1, it follows $R \cdot \widehat{\gamma_{\Lambda}}=\widehat{R \cdot \gamma_{\Lambda}}=\widehat{\gamma_{\Lambda}}$ for all rotations $R$. Altogether we have obtained the following result.

Theorem 3.3. Let $\Lambda$ be as in Theorem 3.1 arising from a tiling with statistical circular symmetry. Then the diffraction of $\Lambda$ is circular symmetric.

An immediate consequence is that the pure point part of the diffraction spectrum of a tiling with statistical circular symmetry is at most one peak at the origin. A simple argument shows that this peak always exists, compare [9, [12]. These results are relevant for physics because such tilings can serve as a simple model for powder diffraction [3].

\section{Tiling Dynamical Systems}

An important tool in investigating properties of nonperiodic tilings is the fact that such a tiling gives rise to a dynamical system, compare [15], [20, [18], [4], [16]. From the properties of 
the dynamical system one can deduce properties of the tiling. For brevity, we describe the metrical dynamical system corresponding to [14]. In the context of this paper, the topology defined by this metric is the same as the more general local rubber topology in [4, as well as the topology in [15] and the local topology in [18]. Let $d$ be the following metric, measuring whether two tilings are 'close' to each other.

$$
d\left(\mathcal{T}, \mathcal{T}^{\prime}\right)=\min \left\{\frac{1}{\sqrt{2}}, \inf _{\varepsilon>0}\left\{\varepsilon: B_{1 / \varepsilon} \cap(\mathcal{T}+s)=B_{1 / \varepsilon} \cap\left(R_{\alpha} \mathcal{T}^{\prime}+t\right),\|s\|,\|t\| \leq \frac{\varepsilon}{2},|\alpha| \leq \varepsilon\right\}\right\}
$$

If $d\left(\mathcal{T}, \mathcal{T}^{\prime}\right)$ is small, then $\mathcal{T}$ and $\mathcal{T}^{\prime}$ agree on a large ball around the origin, after a small rotation followed by a small translation. Now, the hull $\mathbb{X}_{\mathcal{T}}$ of the tiling $\mathcal{T}$ is the closure of $\left\{\mathcal{T}+t: t \in \mathbb{R}^{2}\right\}$ with respect to $d$. It is a well-known fact that the hull of a primitive substitution tiling with substitution $\sigma$ is the family $\mathbb{X}_{\sigma}$ of all substitution tilings (compare Def. 1.1). Therefore, we denote the hull of $\mathcal{T}$ by $\mathbb{X}_{\sigma}$, if it is clear which substitution $\sigma$ belongs to $\mathcal{T}$.

A substitution $\sigma$ gives rise to a dynamical system $\left(\mathbb{X}_{\sigma}, G\right)$, where either $G=\mathbb{R}^{2}$ (all translations in the plane), or $G=E(2)$ (all direct Euclidean motions, i.e., all maps $x \mapsto R x+t$, $R$ a rotation, $t$ a translation vector). The study of such dynamical systems yields deep insight into the diffraction properties of the tilings in $\mathbb{X}_{\sigma}$. A milestone in the mathematical diffraction theory is the result that the diffraction spectrum is pure point if and only if the dynamical spectrum is pure point [9, [20, [18, [11, [4]. Other important results are collected in the following theorem. It summarises contributions of several people to the subject over the last two decades. For brevity, it is formulated for the special case of plane tilings only.

Theorem 4.1. Let $\sigma$ be a primitive substitution.

(1) $\mathbb{X}_{\sigma}$ has uniform cluster frequencies, that is, each patch occurs with a well defined frequency throughout the tilings in $\mathbb{X}_{\sigma}$. Here, frequency is defined analogously to (1).

(2) If the tiles in tilings in $\mathbb{X}_{\sigma}$ show finitely many orientations, then $\left(\mathbb{X}_{\sigma}, \mathbb{R}^{2}\right)$ is uniquely ergodic.

(3) If the tiles in tilings in $\mathbb{X}_{\sigma}$ show infinitely many orientations, then $\left(\mathbb{X}_{\sigma}, G\right)$ is uniquely ergodic, where $G \in\left\{\mathbb{R}^{2}, E(2)\right\}$.

(4) All tilings in $\mathbb{X}_{\sigma}$ have the same autocorrelation, thus the same diffraction spectrum.

The first part is a simple consequence of Def. 1.1, compare the discussion in Section 2, or [20] for 'self-affine' tilings. (Each $\mathbb{X}_{\sigma}$ as above contains a self-affine tiling, and by primitivity, all tilings in $\mathbb{X}_{\sigma}$ have the same cluster frequencies.) However, for tilings with statistical circular symmetry, these frequencies are zero. But see [6] for a similar uniformity result in this case. A detailled proof of the second part can be found in [20] or [11]. For tilings with tiles in infinitely many orientations, part three is stated in 14 for $G=E(2)$. For $G=\mathbb{R}^{2}$, it can hopefully be shown via Theorem 2.2, adapting methods from [11]. We aim to give a precise and detailled proof in the future. The last part of the theorem is an immediate consequence from part one and the definition of the autocorrelation.

\section{ACKNowledGements}

It is a pleasure to thank Christoph Richard for many helpful comments. This work was supported by the German Research Council (DFG), within the CRC 701. 


\section{REFERENCES}

[1] M. Baake, Mathematical Diffraction in Euclidean Spaces, lecture notes, EPFL Lausanne (2004).

[2] M. Baake, D. Frettlöh: SCD patterns have singular diffraction, J. Math. Phys. 46 (2005) 033510; math-ph/0411052

[3] M. Baake, D. Frettlöh and U. Grimm, A radial analogue of Poisson's summation formula with applications to powder diffraction and pinwheel patterns, J. Geom. Phys. 57 (2007) 1331-1343; math/0610408

[4] M. Baake and D. Lenz, Dynamical systems on translation bounded measures: Pure point dynamical and diffraction spectra, Ergodic Th. \& Dynam. Syst. 24, 1867-1893 (2004); math/0302231.

[5] D. Frettlöh: Duality of model sets generated by substitutions, Rev. Roumaine Math. Pures Appl. 50 (2005) 619-639; math/0601064.

[6] D. Frettlöh, Substitution tilings with statistical circular symmetry, European J. Combin., accepted; math/0704.2521.

[7] D. Frettlöh and E. Harriss, Tilings Encyclopedia, available online at: http://tilings.math.uni-bielefeld.de

[8] C. Goodman-Strauss, Matching Rules and Substitution Tilings, Annals of Mathematics 147 181-223 (1998).

[9] A. Hof, On diffraction by aperiodic structures, Commun. Math. Phys. 169, 25-43 (1995).

[10] J. C. Lagarias, Mathematical quasicrystals and the problem of diffraction, in: Directions in Mathematical Quasicrystals, eds. M. Baake and R. V. Moody, CRM Monograph Series, vol.13, AMS, Providence, RI (2000) pp. 61-93.

[11] J.-Y. Lee, R.V. Moody and B. Solomyak, Pure point dynamical and diffraction spectra, Ann. Henri Poincaré 3 1003-1018 (2002) mp_arc/02-39.

[12] R.V. Moody, D. Postnikoff and N. Strungaru, Circular symmetry of pinwheel diffraction, Ann. H. Poincaré 7, 711-730 (2006).

[13] N. Pytheas Fogg, Substitutions in Dynamics, Arithmetics and Combinatorics, Lecture Notes in Mathematics 1794, Springer, Berlin (2002).

[14] C. Radin, Space tilings and substitutions, Geometriae Dedicata 55 257-264 (1995).

[15] C. Radin and M. Wolff, Space tilings and local isomorphism, Geom. Dedicata 42, 355-360 (1992).

[16] E. A. Robinson Jr, Symbolic dynamics and tilings of $\mathbb{R}^{d}$, in: Symbolic dynamics and its applications, Proc. Sympos. Appl. Math. 60, AMS, Providence, RI (2004) pp. 81-119.

[17] W. Rudin: Functional Analysis, 2nd ed., McGraw-Hill (1991).

[18] M. Schlottmann, Generalized model sets and dynamical systems, in: Directions in Mathematical Quasicrystals, eds. M. Baake and R. V. Moody, CRM Monograph Series, vol.13, AMS, Providence, RI (2000) pp. 143-159.

[19] L. Schwartz, Théorie des Distributions, rev. ed., Hermann, Paris (1998)

[20] B. Solomyak: Dynamics of self-similar tilings, Ergodic Theory Dynam. Systems 17 (1997) 695-738.

B. Solomyak: Corrections to "Dynamics of self-similar tilings", Ergodic Theory Dynam. Systems 19 (1999) 1685 .

Fakultät Für Mathematik, Bielefeld, Germany

E-mail address: dirk.frettloeh@math.uni-bielefeld.de

$U R L:$ http://www.math.uni-bielefeld.de/baake/frettloe 